\title{
Clinical utility gene card: for pseudoxanthoma elasticum
}

\author{
Anne Legrand $\mathbb{D}^{1,2,3} \cdot$ Karelle Benistan ${ }^{4} \cdot$ Jean Michael Mazzella ${ }^{1}$ Salma Adham ${ }^{1} \cdot$ Michael Frank ${ }^{1} \cdot$ \\ Xavier Jeunemaitre ${ }^{1,2,3} \cdot$ Juliette Albuisson $^{1,2,3}$
}

Received: 10 August 2017 / Revised: 21 November 2017 / Accepted: 5 December 2017 / Published online: 27 February 2018

(c) European Society of Human Genetics 2018

\section{Disease characteristics}

\subsection{Name of the disease (synonyms)}

Pseudoxanthoma elasticum (PXE), Grönblad-Strandberg syndrome.

\subsection{OMIM\# of the disease}

264800

\subsection{Name of the analyzed genes or DNA/ chromosome segments}

ABCC6 located in 16p13.11 chromosomal segment. The corresponding protein is also named Multidrug ResistanceAssociated Protein 6; MRP6.

\subsection{OMIM\# of the gene(s)}

603234

$\triangle$ Anne Legrand

anne.legrand@aphp.fr

1 Département de Génétique, Service de Médecine Vasculaire et Centre de Référence des Maladies Vasculaires Rares, AP-HP, Hôpital Européen Georges Pompidou, Paris, France

2 Paris centre de Recherche Cardiovasculaire-PARCC, INSERM, U970, Paris, France

3 Faculté de Médecine, Université Paris Descartes, Sorbonne Paris Cité, Paris, France

4 Département de Génétique, AP-HP, Hôpital Raymond Poincaré, Garches, France

\subsection{Mutational spectrum}

More than 300 distinct pathogenic variants have been reported in the $A B C C 6$ gene [1]. Most of these variants are gathered in the public $A B C C 6$ variants database (https://data bases.lovd.nl/shared/genes/ABCC6). These include missense, nonsense, frameshift, splice-site single-nucleotide variants (SNVs), and small deletions/insertions. In addition, large deletions or duplications of all or part of the gene are also frequently identified. Pathogenic variants are responsible for a partial or complete loss-of-function.

Most variants are private, although two recurrent variants have been described. The SNV c.3421 C > T, p. (Arg1141Ter) is found with a prevalence of $25 \%$ in various ethnic backgrounds, and a large deletion of $>16 \mathrm{~kb}$ encompassing exons 23-29 (c.2996-1724_4209-478del, also called "del23-29") has a prevalence of $28 \%$ in Americans of European descent [2] and $11 \%$ in Caucasians [1]. Few other variants are found with a low level of recurrence.

Causative variants are distributed throughout the gene, but two intracellular domains are significantly enriched with missense variants: the eighth intracellular loop and the nucleotide binding fold [1].

Polymorphic variants are listed in the dbSNP Database (http://www.ncbi.nlm.nih.gov/snp/), the NHLBI Exome Sequencing Project (http://evs.gs.washington.edu/EVS/), and in the Genome Aggregation Database (http://gnomad. broadinstitute.org). Please note that the abovementioned databases include pathogenic variants.

\subsection{Analytical methods}

The analysis should at least combine Sanger sequencing and an analysis of the recurrent deletion del23-29.

Sanger sequencing targets the 31 coding exons and flanking intronic sequences of the $A B C C 6$ gene (NCBI reference sequence: NG_007558.2, NM_001171.5). Specific primers are needed to avoid amplification of the very similar ABCC6 pseudogenes $\Psi 1$ and $\Psi 2$ sequences [3]. 
The del23-29 can be analyzed with a published method using multiplex long-range PCR [4] or by multiplex ligation-dependent probe amplification (MLPA).

MLPA or any other technique targeting genetic imbalances of $A B C C 6$ exons should be systematically performed in cases with single heterozygous point variants or suspected hemizygosity based on sequencing results. However, MLPA lacks coverage for several ABCC6 exons (exons 1, 3, 6, 16, 19, 20, 29, and 31).

Combining Sanger sequencing with multiplex PCR and MLPA identifies variants in $\sim 90 \%$ of patients. Indeed, deep intronic variants, large deletions, and duplications including exons not explored by MLPA would not be detected using this strategy.

\subsection{Analytical validation}

Sequencing of both DNA strands for SNVs. Diseasecausing variants should be confirmed using genomic DNA from an independent sample.

When an unknown variant (UV) is identified, analytical validation should systematically integrate interspecies conservation analysis of the mutant nucleotide(s) and amino$\operatorname{acid}(\mathrm{s})$, as well as the surrounding sequence(s). The identified variant(s) should systematically be searched in polymorphisms and disease variants databases.

\subsection{Estimated frequency of the disease (incidence at birth ("birth prevalence") or population prevalence)}

Estimated population prevalence ranges between 1/25,000 and $1 / 50,000$ [5].

Incidence of de novo pathogenic variants is very low [1, 6]. All ethnic groups are involved. There is a sex bias toward women (sex ratio 1:2 [1]).

If known to be variable between ethnic groups, please report:

The frequency does not seem to vary between different ethnical backgrounds. However, in consanguinous populations occurrence of new cases could theoretically increase.

\subsection{Diagnostic setting}

\begin{tabular}{llc}
\hline & Yes. & No. \\
A. (Differential) diagnosis & $\bigotimes$ & $\square$ \\
B. Predictive testing & $\bigotimes$ & $\square$ \\
C. Risk assessment in relatives & $\bigotimes$ & $\square$ \\
D. Prenatal & $\square$ & $\square$ \\
\hline
\end{tabular}

Comment: A first comment must be made concerning differential diagnoses. One first differential diagnosis has to be considered in case of severe and early-onset arterial calcifications. This recessive disorder is named generalized arterial calcification of infancy (GACI) and is caused by pathogenic variants in the ENPPI gene encoding ectonucleotide pyrophosphatase/phosphodiesterase 1 . It is characterized by hydroxyapatite deposits in the internal elastic lamina of large and medium-sized arteries associated with intimal proliferation, leading to severe arterial stenoses, and organs' ischemia. Causal variants in ENPP1 are found in approximatively $75 \%$ of cases [7, 8]. Among the remaining $25 \%$, some cases harbor mono- or biallelic $A B C C 6$ pathogenic variants. Hence an important overlap of genotypes and phenotypes shows that GACI and PXE belong to the same clinical spectrum of ectopic calcification [9]. To date, no digenism has been described between these two genes.

The second differential diagnosis is a recessive condition named PXE-like disorder with multiple coagulation factor deficiency (PXE-like) and is caused by pathogenic variants in $G G C X$ encoding $\gamma$-glutamyl carboxylase [10]. PXE-like shares some manifestations with PXE: yellowish papules or leathery plaques associated with fragmentation and calcification of elastic fibers in the dermis, ocular peau d'orange, and/or angiod streaks (AS), and accelerated arteriosclerosis. Differential diagnosis relies on more widespread and severe skin lesions and inconstant association with retinopathy, cardiac malformations, and deficiency of the vitamin-K dependent factors resulting in abnormal bleeding tendency [11]. Cases with PXE-like skin phenotype and no clotting factor deficiency were found to harbor pathogenic variants in both genes, one variant in $G G C X$ and one variant in $A B C C 6$, revealing digenic inheritance [12].

Another differential diagnosis is about angiod streaks, which are not pathognomic of PXE. Indeed hemoglobinopathies, and in particular sickle-cell disease and $\beta$-thalassemia, should be considered.

A second comment is needed concerning the family screening. Identifying two pathogenic variants in a patient enables to test the siblings. Asymptomatic parents are expected to have transmitted one pathogenic variant each, and their screening is not relevant. Few cases of pseudodominant transmission have been described and were proven to occur in families where three mutations co-segregate $[1,13]$. In these latter cases, the parents are tested to properly define inheritance of the variants.

A third comment relates to prenatal diagnosis. A prenatal test is technically feasible. However, in most cases PXE is not a life-threatening condition and is neither responsible for major disability. Thus pregnancy termination is not required. 


\section{Test characteristics}

$\begin{array}{ll}\begin{array}{l}\text { Genotype or A:true positives } \\ \text { disease }\end{array} & \begin{array}{l}\text { C: false } \\ \text { negative }\end{array} \\ \text { Present Absent B:false positives } & \begin{array}{l}\text { D:true } \\ \text { negative }\end{array}\end{array}$

Test

\begin{tabular}{llll} 
Pos. A & B & Sensitivity: specificity: & A/(A+C) \\
& & & D/(D+B) \\
Neg. C & D & $\begin{array}{l}\text { Pos. predict. value: } \\
\text { Neg. predict. value: }\end{array}$ & A/(A+B) \\
& & D/C $(\mathrm{C}+\mathrm{D})$ \\
\hline
\end{tabular}

\subsection{Analytical sensitivity}

(proportion of positive tests if the genotype is present)

$100 \%$. If only one mutation is identified by the combination of Sanger sequencing and multiplex PCR targeting the recurrent deletion del23-29, the analysis should be completed by MLPA to detect other large rearrangements. Unfortunately only 23 of the 31 exons of ABCC6 are investigated by MLPA. In case of obvious clinical diagnosis and a negative MLPA result, a high-density array-CGH or another semi-quantitative technique covering the 31 exons should also be performed.

\subsection{Analytical specificity}

(proportion of negative tests if the genotype is not present)

Analytical specificity reaches nearly $100 \%$. False positive results are rare and could be explained by misinterpreting known or unknown variants: some SNV were historically classified as pathogenic, and should be reclassified as polymorphism due to their frequency in the general population (for example, p.(Arg391Gly) minor allele frequency is $0.8 \%$ in Caucasians but was initially reported pathogenic [3]). ABCC6 was found to have a role in hepatic ATP release and then its conversion in inorganic pyrophosphate (PPi), a key inhibitor of ectopic mineralization [14]. However, no functional test (e.g., measurement of plasma PPi level) is available to confirm or deny the pathogenic effect of UV.

\subsection{Clinical sensitivity}

(proportion of positive tests if the disease is present)

The clinical sensitivity can be dependent on variable factors such as age or family history. In such cases, a general statement should be given, even if a quantification can only be made case by case.
Clinical sensitivity theoretically reaches $100 \%$. A series published in 2017 raises clinical sensitivity to $82 \%$ for all clinical PXE cases and points out that only one pathogenic variant is identified in some cases with uncomplete or atypical phenotype (harboring only skin or ocular manifestations) [1].

Two hypotheses are proposed to explain these results. Heterozygous carriers might display slight skin or eye manifestations [15], which could correspond to a milder phenotype in carriers. Alternatively, the second causal variant could be a non-coding variant not detected by current analysis methods [1].

Inherited disorders close to PXE, like GACI, related to $E N P P 1$ deficiency, or PXE-like, related to $G G C X$ deficiency, could also explain incomplete $A B C C 6$ genotypes. Taking into account the clinical overlap between these conditions and PXE, molecular screening of these two genes should be performed in patients with no or one $A B C C 6$ variant.

\subsection{Clinical specificity}

(proportion of negative tests if the disease is not present)

The clinical specificity can be dependent on variable factors, such as age or family history. In such cases, a general statement should be given, even if a quantification can only be made case by case.

Clinical specificity is nearly $100 \%$ in adults. Indeed, prevalence of skin and eye symptoms increases with age [16].

\subsection{Positive clinical predictive value}

(life-time risk to develop the disease if the test is positive)

Non-penetrance in adulthood is rare and positive predictive value is close to $100 \%$. The clinical severity increases with age and is unpredictable due to intra-familial and inter-familial variability.

\subsection{Negative clinical predictive value}

(probability not to develop the disease if the test is negative)

Assume an increased risk based on family history for a non-affected person. Allelic and locus heterogeneity may need to be considered.

Index case in that family had been tested and two causative variants identified:

$100 \%$. If the non-affected relative does not carry the two causative variants or carries only one variant, he or she has no risk to develop the disease.

Index case in that family had not been tested:

The theoretical recurrence risk is of $25 \%$ for the siblings due to recessive inheritance. 


\section{Clinical utility}

\section{1. (Differential) diagnostics: The tested person is clinically affected}

(To be answered if in 1.9 "A" was marked)

\subsubsection{Can a diagnosis be made other than through a genetic test?}

\begin{tabular}{lll}
\hline No. & $\square$ (continue with 3.1.4) \\
Yes, & $\square$ \\
& clinically. & \\
& imaging. & $\square$ \\
& endoscopy. & $\square$ \\
& biochemistry. & $\square$ \\
& electrophysiology. & $\square$ \\
& other (please describe): & Histopathology.
\end{tabular}

\subsubsection{Describe the burden of alternative diagnostic methods to the patient}

Clinical diagnosis (combining family history and physical examination) associated with histopathological analysis of a skin biopsy is the only alternative method.

As the combination of skin and eye typical lesions is present in most cases with one or two variants [1], both organs must be evaluated by expert clinicians in dermatology and ophtalmology. Skin examination can reveal yellowish papules in the flexural locations or coalescent plaques, which rarely affect non-flexural sites. Histopathological skin analysis confirms the diagnosis and shows an increase and fragmentation of elastin fibers with typical clumping and calcification on light microscopy and von Kossa staining.

PXE ocular signs, peau d'orange, and angioid streaks in the retina are detected by fundus. They are sometimes associated with complications: retinal hemorrhages and neovascularization.

Cardiovascular and renal complications are rare (10\% of cases). Their evaluation is generally used for severity assessment but not for clinical diagnosis.

\subsubsection{How is the cost-effectiveness of alternative diagnostic methods to be judged?}

Physical examination, skin biopsy, and fundus are costeffective, compared to genetic screening. However, clinical diagnosis does not help for screening the at-risk relatives, particularly in young adults and children.
3.1.4. Will disease management be influenced by the result of a genetic test?

No.

Yes. $\otimes$

Therapy

(please

describe)

Prognosis

(please

describe)

There is no specific treatment for PXE. The treatment depends on clinical manifestations ( $c f$ Management).

Genotype-phenotype correlation shows a more severe eye and vascular phenotype in patients with total lossof-function variants. This implies an enhanced follow-up based on fundoscopy and vascular imaging.

Management The current management of PXE is (please multidisciplinary (dermatologist, describe) ophthalmologist, cardiologist, vascular and plastic surgeon, geneticist, nutritionist). The management and treatment depend on clinical manifestations and include: -Eye management: the patients should learn to use the Amsler grid to control for central visual disturbances. Eye protection is needed for high-risk activities. Intravitreal injections of vascular endothelial growth factor (VEGF) antagonists are used to prevent neovascularization in cases of retinal hemorrhages. -Lipid levels should be monitored periodically and treated if necessary (diseasecausing lipid alterations are not linked to PXE but they should be avoided to prevent atherosclerosis and aggravation of existing vascular lesions due to PXE). Endovascular and surgical intervention are required for severe peripheral vascular disease. -Surgical intervention can be necessary for gastrointestinal bleeding. -Plastic surgery is sometimes used for redundant skin reduction. -Other treatments are currently being assessed: magnesium intake, phosphate binders, and bisphosphonates such as etidronate (bisphosphonates are pyrophosphate analogs, they are used to treat ectopic mineralization thanks to their antimineralization and antiosteoclastic activities) [17]. 


\subsection{Predictive setting: The tested person is clinically unaffected but carries an increased risk based on family history}

(To be answered if in 1.9 "B" was marked)

\subsubsection{Will the result of a genetic test influence lifestyle and prevention?}

If the test result is positive (please describe):

Dietary and lifestyle modifications are needed to delay or prevent complications. Cardiovascular risk factor (particularly overweight, hypertension, lipid disorders, and smoking) prevention and treatment is essential: smoking cessation, weight loss, daily walking, moderate physical exercise, etc. Aspirin and other non-steroidal anti-inflammatory medications should be avoided because of gastrointestinal hemorrhage risk. Contact sports without appropriate eye and head protection should also be avoided to prevent eye trauma that could cause retinal hemorrhage. Magnesium supplementation could be also proposed to PXE patients to prevent connective tissue mineralization (the benefits observed in mice have to be proven in humans) [18].

If the test result is negative (please describe):

No.

\subsubsection{Which options in view of lifestyle and prevention} does a person at-risk have if no genetic test has been done (please describe)?

Lifestyle and prevention should be exactly the same as above if the clinical diagnosis is certain.

\subsection{Genetic risk assessment in family members of a diseased person}

(To be answered if in 1.9 "C" was marked)

3.3.1. Does the result of a genetic test resolve the genetic situation in that family?

Genetic testing is necessary for genetic risk assessment in siblings.

\subsubsection{Can a genetic test in the index patient save genetic or} other tests in family members?

Not applicable

\subsubsection{Does a positive genetic test result in the index} patient enable a predictive test in a family member?

Family screening can be performed after identification of two causal variants in the index case.

\subsection{Prenatal diagnosis}

(To be answered if in 1.9 "D" was marked)

3.4.1. Does a positive genetic test result in the index patient enable a prenatal diagnosis?

Not applicable.

\section{If applicable, further consequences of testing}

Please assume that the result of a genetic test has no immediate medical consequences. Is there any evidence that a genetic test is nevertheless useful for the patient or his/her relatives? (Please describe)

The genetic test confirms the clinical diagnosis of PXE and allows genetic counselling, family screening, and prevention of complications. For example, prevention of eye trauma with adapted protection in high-risk activities can be proposed to the concerned relatives.

Acknowledgements This work was supported by EuroGentest2 (Unit 2: "Genetic testing as part of health care"), a Coordination Action under FP7 (Grant Agreement Number 261469), and the European Society of Human Genetics.

\section{Compliance with Ethical Standards}

Conflict of interest The authors declare that they have no conflict of interest.

\section{References}

1. Legrand A, Cornez L, Samkari W, et al. Mutation spectrum in the ABCC6 gene and genotype-phenotype correlations in a French cohort with pseudoxanthoma elasticum. Genet Med. 2017;19:909-917.

2. Le Saux O, Beck K, Sachsinger C, et al. A spectrum of ABCC6 mutations is responsible for pseudoxanthoma elasticum. Am J Hum Genet. 2001;69:749-764.

3. Pulkkinen L, Nakano A, Ringpfeil F, Uitto J. Identification of ABCC6 pseudogenes on human chromosome 16p: implications for mutation detection in pseudoxanthoma elasticum. Hum Genet. 2001;109:356-365.

4. Ringpfeil F, Nakano A, Uitto J, Pulkkinen L. Compound heterozygosity for a recurrent 16.5-kb Alu-mediated deletion mutation and single-base-pair substitutions in the ABCC6 gene results in pseudoxanthoma elasticum. Am J Hum Genet. 2001;68:642-652.

5. Prunier F, Terrien G, Le Corre Y, et al. Pseudoxanthoma elasticum: cardiac findings in patients and Abcc6-deficient mouse model. PLoS ONE. 2013;8:e68700.

6. Bergen AA, Plomp AS, Schuurman EJ, et al. Mutations in ABCC6 cause pseudoxanthoma elasticum. Nat Genet. 2000;25:228-231.

7. Ruf N, Uhlenberg B, Terkeltaub R, Nürnberg P, Rutsch F. The mutational spectrum of ENPP1 as arising after the analysis of 23 
unrelated patients with generalized arterial calcification of infancy (GACI). Hum Mutat. 2005;25:98.

8. Rutsch F, Böyer P, Nitschke Y, et al. Hypophosphatemia, hyperphosphaturia, and bisphosphonate treatment are associated with survival beyond infancy in generalized arterial calcification of infancy. Circ Cardiovasc Genet. 2008;1:133-140.

9. Nitschke Y, Baujat G, Botschen U, et al. Generalized arterial calcification of infancy and pseudoxanthoma elasticum can be caused by mutations in either ENPP1 or ABCC6. Am J Hum Genet. 2012;90:25-39.

10. Vanakker OM, Martin L, Gheduzzi D, et al. Pseudoxanthoma elasticum-like phenotype with cutis laxa and multiple coagulation factor deficiency represents a separate genetic entity. J Invest Dermatol. 2007;127:581-587.

11. De Vilder EYG, Debacker J, Vanakker OM. GGCX-associated phenotypes: an overview in search of genotype-phenotype correlations. Int J Mol Sci. 2017;18:E240.

12. Li Q, Schurgers LJ, Smith ACM, Tsokos M, Uitto J, Cowen EW. Co-existent pseudoxanthoma elasticum and vitamin K-dependent coagulation factor deficiency: compound heterozygosity for mutations in the GGCX gene. Am J Pathol. 2009; 174:534-540.
13. Ringpfeil F, McGuigan K, Fuchsel L, et al. Pseudoxanthoma elasticum is a recessive disease characterized by compound heterozygosity. J Invest Dermatol. 2006;126:782-786.

14. Jansen RS, Duijst S, Mahakena S, et al. ABCC6-mediated ATP secretion by the liver is the main source of the mineralization inhibitor inorganic pyrophosphate in the systemic circulation-brief report. Arterioscler Thromb Vasc Biol. 2014;34:1985-1989.

15. Martin L, Maître F, Bonicel P, et al. Heterozygosity for a single mutation in the ABCC6 gene may closely mimic PXE: consequences of this phenotype overlap for the definition of PXE. Arch Dermatol. 2008;144:301-306.

16. Plomp AS, Toonstra J, Bergen AAB, van Dijk MR, de Jong PT. Proposal for updating the pseudoxanthoma elasticum classification system and a review of the clinical findings. Am J Med Genet A. 2010;152A:1049-1058.

17. Uitto J, Jiang Q, Váradi A, Bercovitch LG, Terry SF. Pseudoxanthoma elasticum: diagnostic features, classification, and treatment options. Expert Opin Orphan Drugs. 2014;2:567-577.

18. Li Q, Larusso J, Grand-Pierre AE, Uitto J. Magnesium carbonatecontaining phosphate binder prevents connective tissue mineralization in Abcc6(-/-) mice-potential for treatment of pseudoxanthoma elasticum. Clin Transl Sci. 2009;2:398-404. 